\title{
VALIDAÇÃO EXPERIMENTAL DE SIMULAÇÕES CFD DE UM LEITO FLUIDIZADO CIRCULANTE GÁS-SÓLIDO TIPO RISER
}

\author{
H. J. B. de LIMA FILHO ${ }^{1}$, M. F. P. de BRITO ${ }^{3}$, M. BENACHOUR ${ }^{2}$, V. A. SANTOS ${ }^{1}$, C. C. \\ DANTAS $^{3}$ \\ ${ }^{1}$ Universidade Católica de Pernambuco - Centro de Ciências e Tecnologia (CCT) \\ ${ }^{2}$ Universidade Federal de Pernambuco, Departamento de Engenharia Química (DEQ) \\ ${ }^{3}$ Universidade Federal de Pernambuco, Departamento de Energia Nuclear (DEN) \\ E-mail para contato: hilario@unicap.br
}

\begin{abstract}
RESUMO - O presente trabalho propõe uma modelagem tridimensional do escoamento ascendente das fases fluida e particulada de um riser, em uma unidade piloto à frio (UPF), semelhante à um leito fluidizado circulante (CFB), por meio da aplicação da fluidodinâmica computacional (CFD). Reatores tipo CFB são amplamente utilizados em processo de FCC (craqueamento catalítico fluido) na industria do refino de petróleo, sobretudo em petróleos pesados de cadeia longa. O riser é o local onde ocorrem as reações que dão origem aos produtos de alto valor agregado, e seu desepenho dependente das características hidrodinâmicas do leito. Estudou-se diferentes condições de fluxo mássico de catalisador e velocidade superficial do gás em um riser construído em material transparente, com $5.64 \mathrm{~m}$ de altura e $0.092 \mathrm{~m}$ de diâmetro. Os resultados das simulações foram comparados com os dados experimentais de velocidade e fração volumétrica de sólidos, obtidos a partir métodos não-invasivos, por meio de transmissão de radiação gama. As medidas experimentais foram realizadas ao longo de diferentes posições axiais na UPF. As frações volumétricas e velocidade do catalisador foram obtidas em seções de teste que possuem fontes de amerício (Am-241) e detector blindados, posicionadas axialmente à $0.650,1.384$ e 3.424 metros. O detector utilizado foi um cintilador de $\mathrm{NaI}(\mathrm{Tl})$ de 2" x 2". A modelagem foi capaz de prever e representar as principais características do escoamento em regime de fluidização rápida.
\end{abstract}

\section{INTRODUÇÃO}

O craqueamento fluido catalítico (FCC) é uma das operações unitárias mais importantes no processo de refino de petróleo, onde os fluidos de petróleo pesados de baixo valor agregado, como o gasóleo de vácuo (VGO) ou resíduo atmosférico são convertendos hidrocarbonetos mais leves como a gasolina e o gás liquefeito de petróleo (Farah, 2012). A unidade de FCC depende, principalmente, da circulação um catalisador zeolítico a elevada temperatura no riser, reator do tipo tubular onde ocorrem as reações, e que transportam o produto craqueado para o reator-separador no qual o catalisador é separado da carga craqueada que segue para uma coluna de destilação para separação dos produtos desejados. O diâmetro médio das partículas do catalisador encontra-se entre 40 e $80 \mu \mathrm{m}$, com densidade de 1000 a $1800 \mathrm{~kg} / \mathrm{m}^{3}$, classificadas como grupo A (Geldart) (Gidaspow, 1994). 


\section{9 a 22 de outubro de 2014 \\ Florianópolis/SC}

A operação de processos em CFB necessita de estratégias específicas para o domínio dessa tecnologia. Estudos anteriores (Malcus et al., 2000) têm verificado a dificuldade de se modelar um $\mathrm{CFB}$, tendo em vista que diferentes estruturas geométricas do leito, condições operacionais e de material particulado podem produzir diferentes características hidrodinâmicas ao leito. Nas últimas décadas Gayfin et al. (1997) e Sterneus et al. (2000) realizaram estudos experimentais para investigar o comportamento hidrodinâmico do riser com base nos perfis radiais da concentração de gás, enquanto que Subramanya et al. (2005) e Gupta et al. (2003) simularam numericamente o efeito da atomização do gasóleo na região de alimentação para avaliar o desenpendo do riser. Segundo Dantas et al. (2006), técnicas não invasivas com uso de radiação são adequadas para o estudo detalhado do comportamento do escoamento interno em risers, em escala piloto a frio (UPF), permitindo a varredura completa do riser para obtenção dos perfis radiais e axiais da distribuição da fração volumétrica de sólidos. A estratégia utilizada é fundamentada na atenuação da radiação gama. Está técnica permite o estudar o escoamento sem pertubar a hidrodinâmica do leito, tendo em vista que as medidas são efetuadas sem inserir instrumentação no interior do reator.

Fundamentado no que foi exposto, o presente trabalho tem como objetivo a validação experimental de simulações fluidodinâmicas computacionais de modelos bifásicos tridimensionais para o escoamento gás-sólido de uma UPF construída em material transparente (acrílico). A simulação CFD foi fundamentada na teroria cinética do escoamento granular, com modelagem Euleriana, implementados no código comercial ANSYS FLUENT versão 15.0. Os dados experimentais foram obtidos por meio de técnicas não-intrusivas, com aplicação de técnicas de atenuação gama.

\section{MATERIAL E MÉTODOS}

\subsection{Descrição do Sistema e Domínio Computacional}

Uma Unidade Piloto a Frio (UPF) foi elaborada e construída basicamente de um sistema do tipo leito fluidizado circulante, onde o catalisador de craqueamento e ar comprimido escoam e dão origem a um fluxo ascendente e diluído em substituição ao escoamento do próprio catalisador, transportado por vapores de gasóleo de vácuo. Catalisador e ar são misturados na base do riser, ascendendo através do mesmo, separando-se em função de uma desaceleração em uma grande câmara no topo desse componente. Dentro da câmara de separação a maior parte do catalisador retorna para a base do sistema, enquanto que o ar comprimido é liberado para o ambiente com auxílio de ciclones e de um filtro de cartucho de papel, semelhante aos utilizados em motores a diesel. A UPF (Figura1) tem suas seções de escoamento confeccionadas em acrílico. O riser, objeto de estudo, possui diâmetros internos iguais a 0,092 m, e comprimento de 5,64 m, assim com a coluna de retorno. O tempo de processamento computacional é fortemente influenciado pelo tamanho e qualidade da malha gerada, tendo em vista que a discretização correta do domínio é um dos principais fatores para solucionar problemas utilizando a metodologia CFD. No presente estudo, foram realizados testes preliminares em uma malha aplicada à uma geometria tridimensional, que representa o domínio físico do riser da UPF, de acordo com a Figura 1 (c), com o objetivo de determinar o número mínimo de células presentes em sua composição, otimizando o processamento computacional das simulações. O número de células determinado nas simulções iniciais equivale a 200000 células, em uma malha estruturada. 
a)

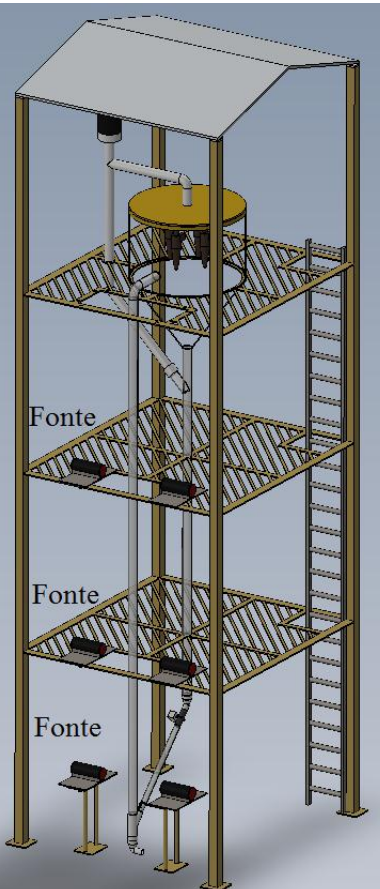

b)

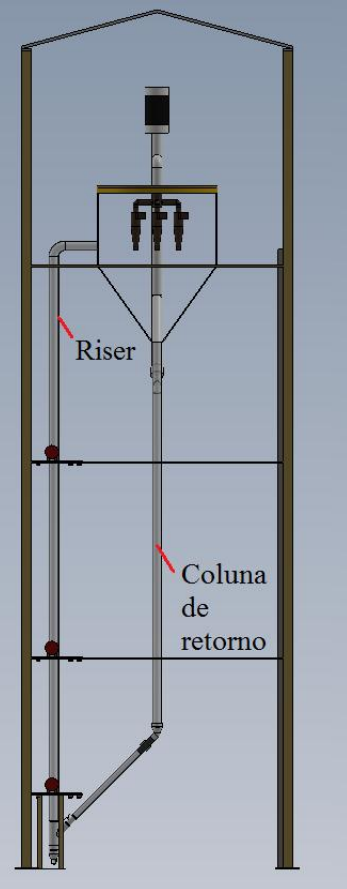

c)

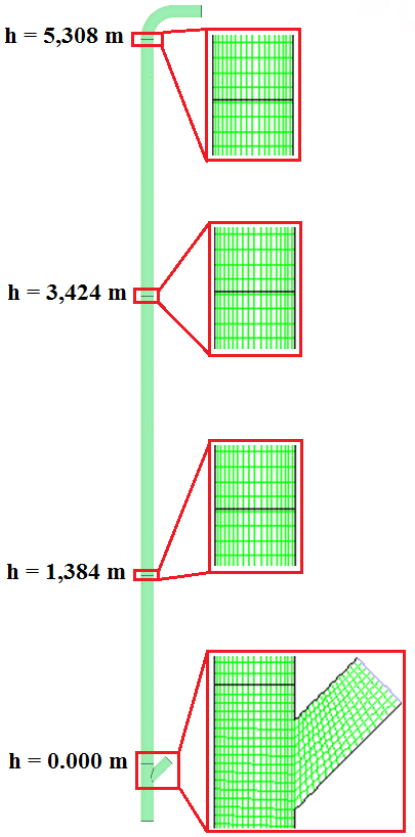

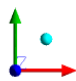

Figura 1 - Unidade piloto à frio: (a) vista isométrica, (b) vista lateral e (c) malha estruturada 3D

\subsection{Arranjo Experimental: Medidas de Transmissão Gama}

As medidas de transmissão gama foram realizadas em três posições axiais no riser $(0,650 \mathrm{~m}$, 1,384 m e 3,424 m), chamadas de seções de testes. Cada seção de teste é formada por uma mesa coordenada, que possuem regulagens para aproximação ou afastamento em relação ao riser. Nesta mesa (Figura 2) estão instalados o detector e a fonte, que possuem blindagem e colimadores com abertura de $5 \mathrm{~mm}$ de diâmetro, e que se movimentam paralelamente por meio de motores de passo controlados por um programa supervisório que permite especificar a distância a ser percorrida, e obtenção dos dados referentes a atenuação gama por meio de fotopicos registrados pelo detector.

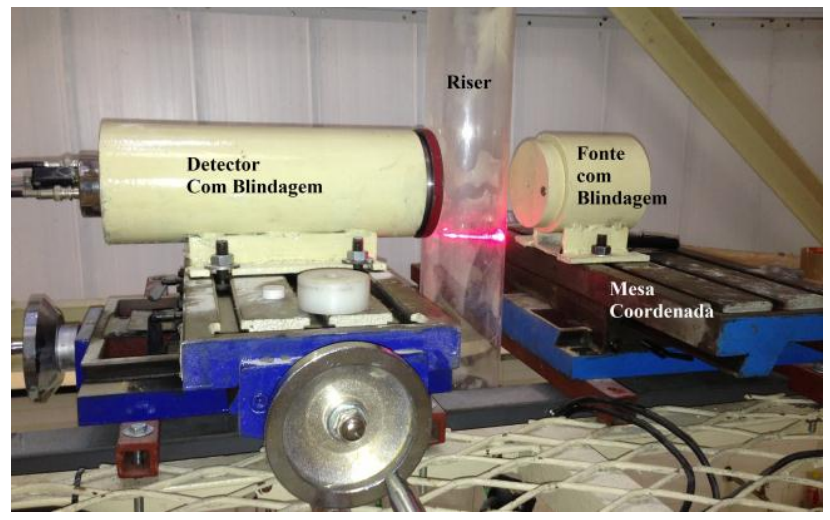

Figura 2 - Seção de testes de transmissão gama: fonte, riser e detector 


\section{9 a 22 de outubro de 2014 \\ Florianópolis/SC}

\subsection{Abordagem aos Modelos Fluididinâmicos Computacionais Multifásicos}

Os modelos matemáticos utilizados para representar escoamentos bifásicos (gás-sólido), atualmente, são baseados em dois tipos básicos de abordagens: a Euleriana e a Lagrangeana. Tendo em vista que a abordagem Lagrangeana é recomendada para sistemas diluídos, utilizou-se a abordagem Euleriana para o presente estudo. Este tipo de abordagem é indicado para sistemas com altas concentrações de partículas.Um conjunto de equações gonvernantes, equações de conservação da massa, momento e constutivas, que serão resolvidas numericamente. As equações utilizadas neste trabalho estão fundamentadas na teoria cinética do escoamento granular, desenvolvido por Gidaspow (1994). Outros pesquisadores comprovaram a validade desta teoria (Neri e Gidaspow, 2000; Sun e Gidaspow, 1999; Andreux et. al., 2007). Mais tarde Yang et. al., (2003) e Yang et. al., (2004) desenvolveram coeficientes de arrasto com base na abordagem da Minimização Multi-Escala (EMMS). Neste trabalho, o modelo de arraste de Gidaspow foi aplicado, tendo nestas condições de fluidização rápida este coeficiente tem apresentado desempenho satisfatório.

\section{RESULTADOS E DISCUSSÃO}

Foram comparados os resultados das simulações CFD com os dados experimentais da fração volumétrica de sólidos (catalisador) em diferentes alturas (posições axiais) ao longo do riser. A Tabela 1 apresenta um resumo das condições de contorno e propriedades físicas empregadas na simulação e ensaio experimental.

Tabela 1 - Condições de contorno e propriedades físicas

\begin{tabular}{|l|c|c|}
\hline & Região de contorno & Propriedades Físicas \\
\hline Entrada de sólidos & $0,06200 \mathrm{~kg} / \mathrm{s}$ & - \\
\hline Entrada de gás & $0,01042 \mathrm{~kg} / \mathrm{s}$ & - \\
\hline Saída (mistura) & $0,07242 \mathrm{~kg} / \mathrm{s}$ & - \\
\hline Parede & não-escorregamento & - \\
\hline Critério de convergência & $10^{-4}$ (residual) & - \\
\hline Coeficiente de arrasto & Gidaspow & - \\
\hline Densidade do gás & - & $1,225 \mathrm{~kg} / \mathrm{m}^{3}$ \\
\hline Densidade do catalisador & - & $900 \mathrm{~kg} / \mathrm{m}^{3}$ \\
\hline Diâmetro da particular & - & $76 \mu \mathrm{m}$ \\
\hline
\end{tabular}

As Figuras 3, 4 e 5 apresentam perfis radiais de frações volumétricas em diferentes alturas. Observa-se maior concentração nas proximidades da parede e base do riser, e diminui no sentido axial positivo. Três regiões distintas são identificadas no riser, formando de gradientes de concentração. A base é a região mais densa, com grandes intesidades turbulentas e concentração assimétrica provocada pela região de entrada de sólidos. Na região de escoamento plenamente desenvolvido observa-se uma região menos densa e aproximadamente simétrica. No topo do riser a concentração de sólidos é muito pequena, com pequeno acúmulo de catalisador nas próximidades da parede, onde observa-se um fluxo de menor intensidade no sentido descendente axial, nas proximidades da parede. 

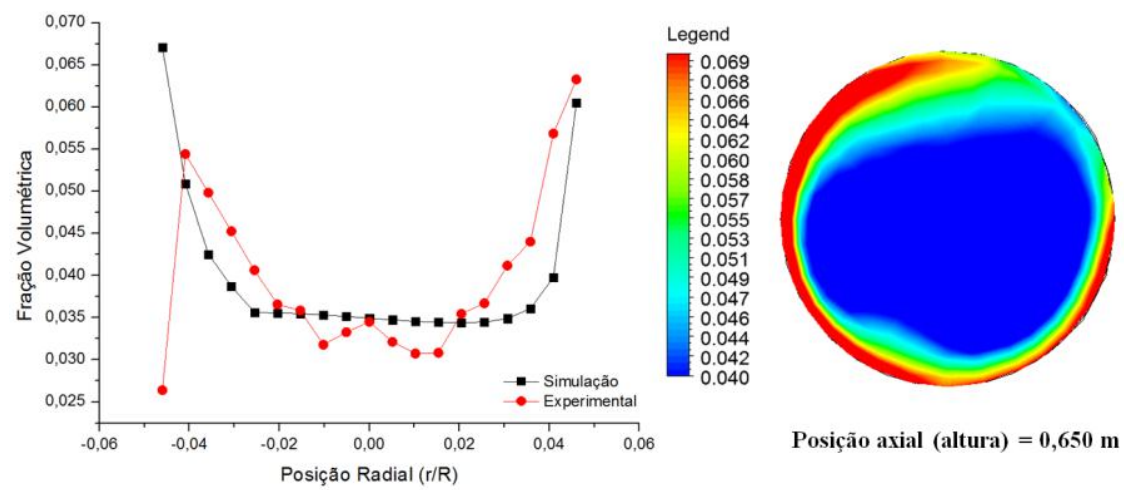

Posição axial (altura) $=0,650 \mathrm{~m}$

Figura 3 - Fração volumétrica de catalisador (posição axial, h = 0,650 m)
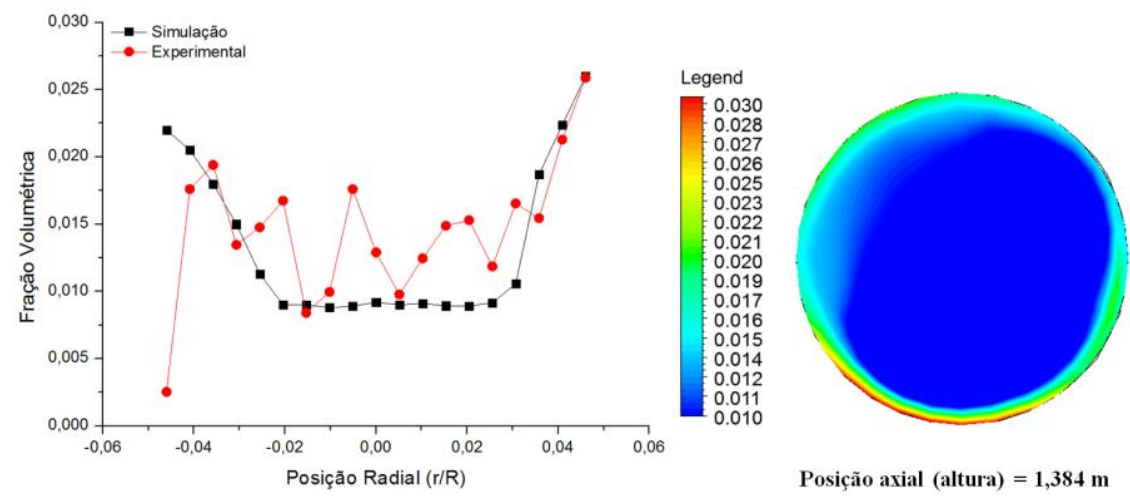

Posição axial (altura) $=1,384 \mathrm{~m}$

Figura 4 - Fração volumétrica de catalisador (posição axial, h = 1,384 m)
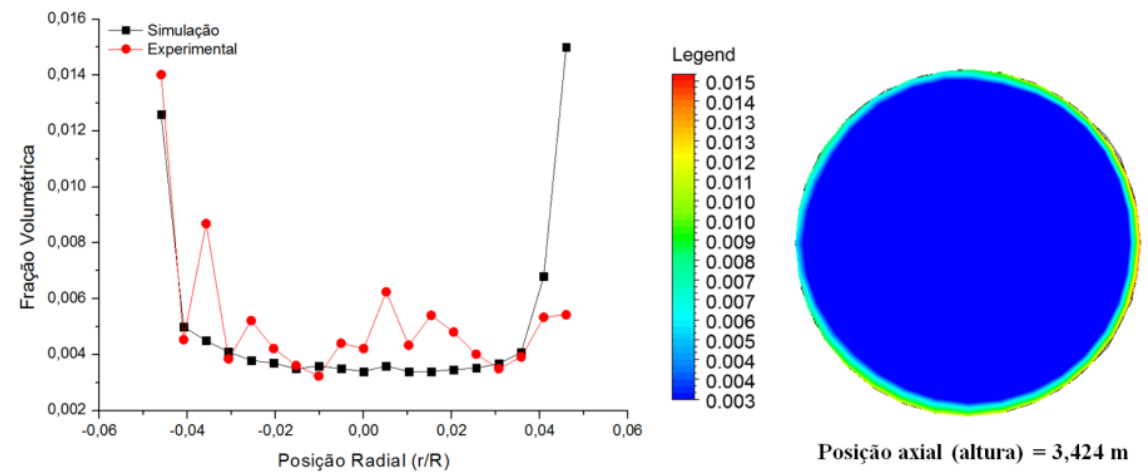

Posição axial (altura) $=3,424 \mathrm{~m}$

Figura 5 - Fração volumétrica de catalisador (posição axial, $\mathrm{h}=0,650 \mathrm{~m}$ )

As Figuras 6, 7 e 8 apresentam perfis de velocidade radial da fase particulada. Observa-se que a velocidade do sólido mantém-se praticamente constante, ao longo do riser, com pequeno decrescimo de velocidade. Verifica-se também que o efeito das colisões entre as partículas e as paredes do riser exercem efeitos significativos em sua hidrodinâmica, apresentando baixas velocidades nas próximidades das paredes. 

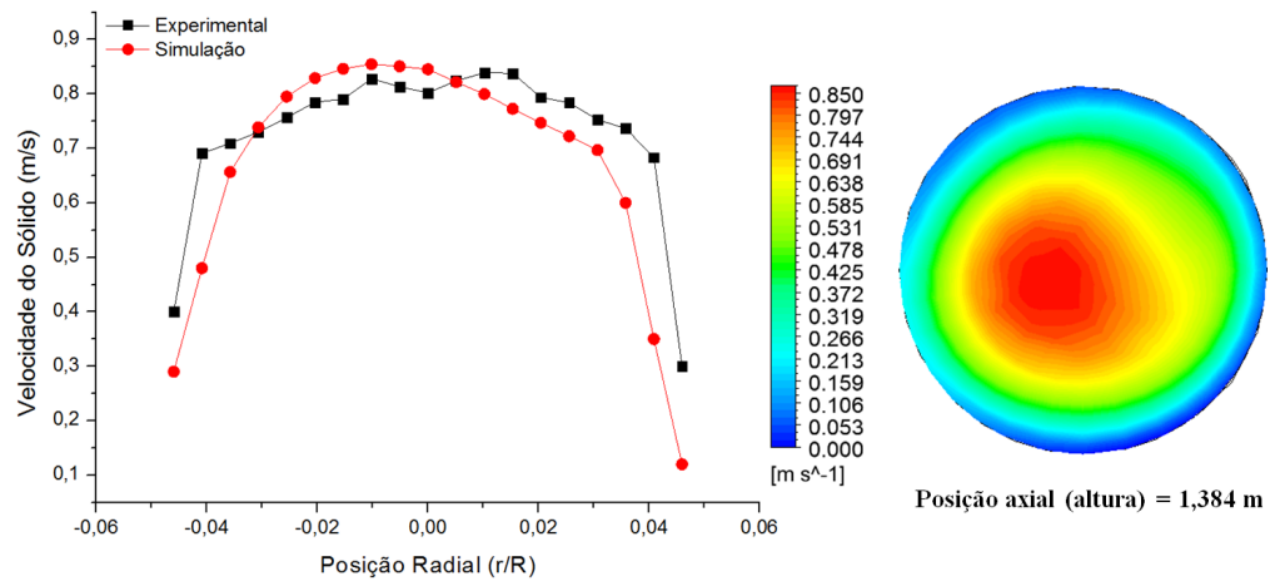

Posição axial (altura) $=1,384 \mathrm{~m}$

Figura 6 - Perfi de velocidade radial da fase particulada (posição axial, $h=1,384 \mathrm{~m}$ )
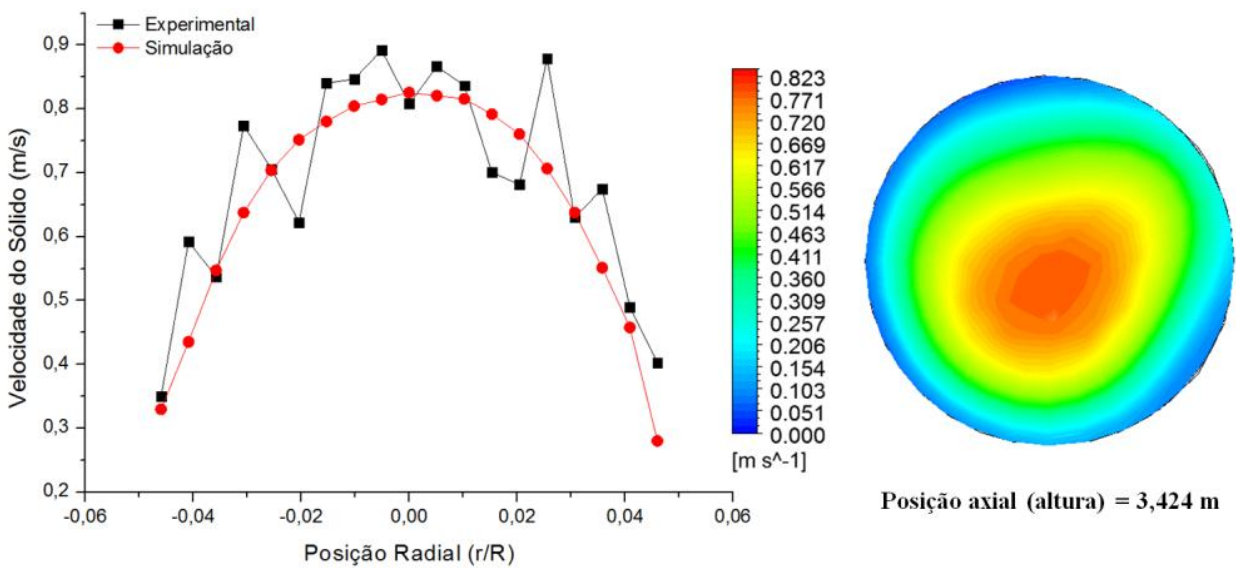

Posição axial (altura) $=3,424 \mathrm{~m}$

Figura 7 - Perfi de velocidade radial da fase particulada (posição axial, $h=3,424 \mathrm{~m}$ )

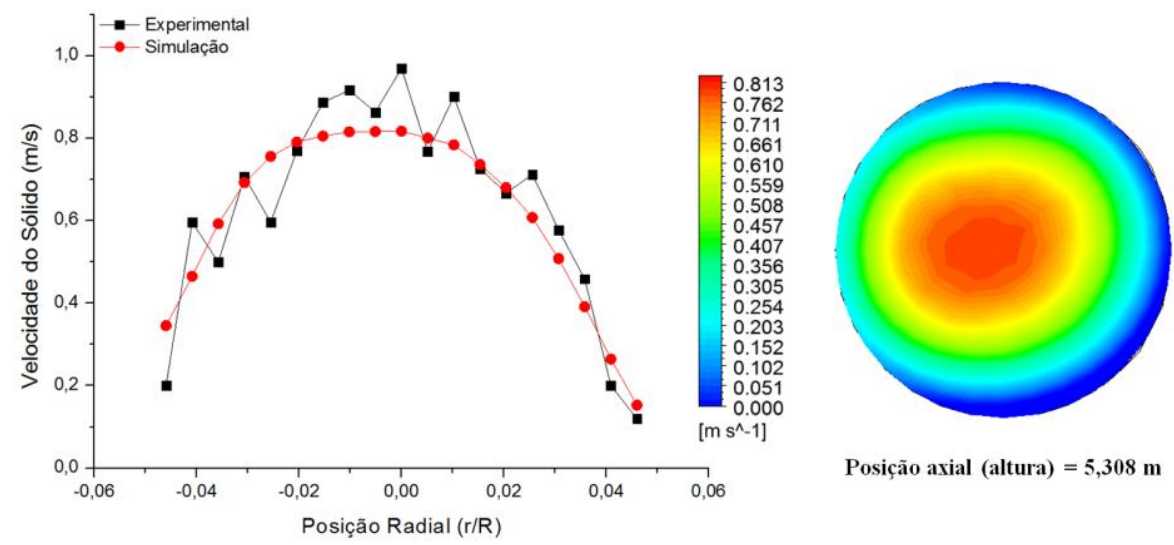

Figura 8 - Perfi de velocidade radial da fase particulada (posição axial, $\mathrm{h}=5,308 \mathrm{~m}$ ) 
A Figura 9 apresenta o resultado da simulação transiente do efeito de diferentes vazões de ar comprimido na região da fase gaosa, mantendo constante o fluxo de catalisador $\left(9,38 \mathrm{~kg} / \mathrm{m}^{2} \mathrm{~s}\right)$, num determinado instante de tempo $(\mathrm{t}=2$ segundos $)$. Verifica-se que maiores vazões resultam na expansão no leito, elevando sua porosidade média, facilmente verificada pela queda de pressão em função de seu comfprimento.

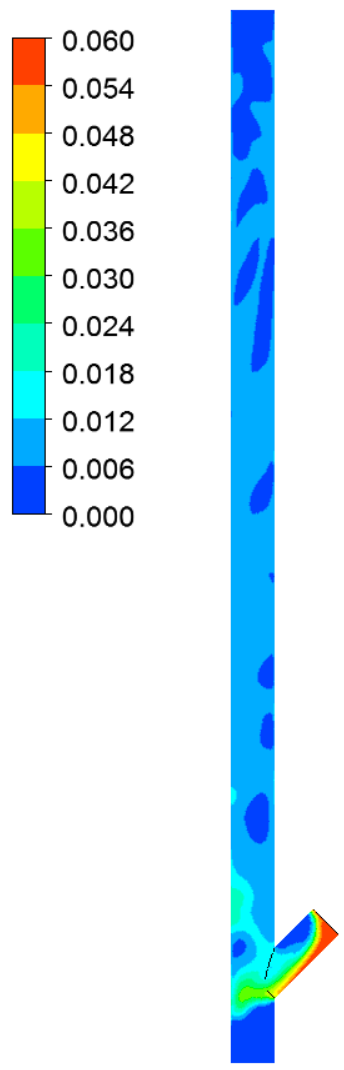

a)

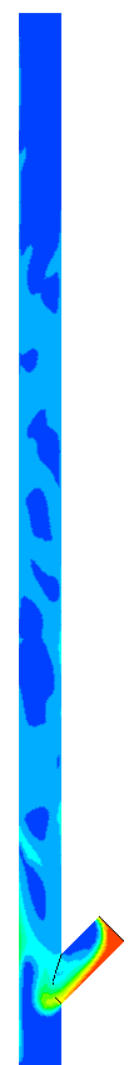

b)

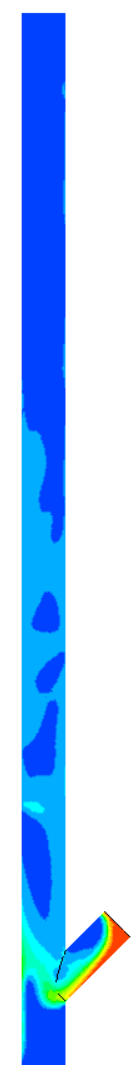

c)

Figura 9 - Efeito da vazão de ar comprimido: a) 700 L/min; b) 600 L/min e c) 500 L/min

\section{CONCLUSÕES}

O objetivo do trabalho foi apresentar a validação dos dados experimentais de uma unidade piloto à frio, aplicando a teoria cinética do escoamento granular na predição do escoamento multifásico em um riser, que apresenta comportamento semelhante a um leito fluidizado circulante, utilizando como ferramenta a fluidodinâmica computacional. Os resultados obtidos por simulações CFD foram coerentes com os dados experimentais, obtidos por gamametria, para a variável fração volumétrica de catalisador, assim como os dados experimentais da queda de pressão, obtidos no riser por meio de sensores distribuídos ao longo do leito também apresentou-se aceitável, mostrando que união de técnicas não-intrusivas e simulações CFD são capazes de predizer com precisão escoamentos complexos como o escoamento gás-sólido, apresentado neste trabalho. 


\section{REFERÊNCIAS}

ANDREUX, R.; PETIT, G.; HEMATI, M.; SIMONIN, O. Hydrodynamic and solid residence time distribution in a circulating fluidized bed: Experimental and 3D computational study. Chem. Eng. Process.v.47, p.463-473, 2007.

DANTAS, C. C.; SANTOS, V. A. ; LIMA, E. A. O. ; MELO, S. B., Measurements of Catalyst Concentration in the Riser of a FCC Cold Model by Gamma Ray Transmission. In: P. Ciarlini; E. Filipe; A. B. Forbes; F. Pavese; C. Perruchet; B. R. L. Siebert. (Org.). Advanced Mathematical and Computational Tools in Metrology VII. VII ed. Singapore: World Scientific Publishing Co. Pte. Ltd. v. 72, p. 284-288, 2006.

FARAH, M. A. Petróleo e Seus Derivados. Rio de Janeiro: LTC, 2012.

GAYFIN, P.; DIEGO, L. F.; ADFINEZ, J. Radial gas mixing in a fast fluidized bed. Powder Technology, v.94, p.163-171, 1997.

GIDASPOW, D. Multiphase Flowand Fluidization: Continuum and Kinetic Theory Description. Boston: Academic Press, 1994.

GUPTA, A.; RAO, D. S. Effect of feed atomization on FCC performance: simulation of entire unit. Chemical Engineering Science, v.58. p.4567-4579, 2003.

HUILIN, L.; GIGASPOW, D.; BOUILLARD, J.; WENTIE, L. Hydrodynamic simulation of gas-solid flow in a riser using kinetic theory of granular flow, Chem. Eng. J. v.95, p.1-13, 2003.

MALCUS, S.; CHAPLIN, G.; PUSLEY, T. The hydrodynamics of the high-density bottom zone in a CFB riser analyzed by means of electrical capacitance tomography (ECT), Chemical Engineering Science, v.55, p.4129-4138, 2000.

NERI, A.; GIDASPOW, D. Riser hydrodynamics: simulation using kinetic theory. AIChE J. v.46, p.52-67, 2000.

STERNEUS, J.; JOHNSSON, F.; LECKNER, B. Gas mixing in circulating fluidized-bed risers. Chemical Engineering Science, v.55, p.129-148, 2000.

SUBRAMANYA, V.; SAKET, L; VIVEK, V. Modeling of vaporization and cracking of liquid oil injected in a gas-solid riser. Chemical Engineering Science, v.60, p.6049-6066, 2005.

SUN, B.; GIDASPOW, D. Computation of circulating fluidized-bed riser flowfor the fluidization, VIII benchmark test, Ind. Eng. Chem. Res. v.38, p.787-792, 1999.

YANG, N.;WANG, W.;GE, W.; LI, J. CFD simulation of concurrent-up gas-solid flow in circulating fluidized beds with structure-dependent drag coefficient. Chem. Eng. J. v.96, p.71-80, 2003.

YANG, N.;WANG, W.;GE, W.; LI, J. Simulation of heterogeneous structure in a circulating fluidized-bed riser by combining the two-fluid model with the EMMS approach. Ind. Eng. Chem. Res. v.43, p.5548-5561, 2004. 